\title{
Núcleos de Segurança do Paciente no Brasil: Um estudo transversal
}

\author{
Patient Safety Centers in Brazill: A cross-sectional study \\ Centros de seguridad del paciente en Brasil: Un estudio transversal
}

Recebido: 27/04/2021 | Revisado: 05/05/2021 | Aceito: 07/05/2021 | Publicado: 21/05/2021

Paula Beatriz Viana

ORCID: https://orcid.org/0000-0002-7460-4347 Universidade Federal de São João del Rei, Brasil E-mail: paula.vianna68@gmail.com

Tássia Luiza Morato Santos

ORCID: https://orcid.org/0000-0002-2974-5386 Universidade Federal de São João del Rei, Brasil E-mail: tassiamorato@ hotmail.com

Helen Ribeiro

ORCID: https://orcid.org0000-0001-9365-7228 Universidade Federal de São João del Rei, Brasil E-mail: helen.cristiny@ufsj.edu.br

Mariana Linhares Pereira

ORCID: https://orcid.org/0000-0002-3214-2789 Universidade Federal de São João del Rei, Brasil E-mail: marianapereira@ufsj.edu.br

Juliano Teixeira Moraes

ORCID: https://orcid.org/0000-0002-1109-962X Universidade Federal de São João del Rei, Brasil E-mail julianotmoraes@ufsj.edu.br

Cristina Sanches

ORCID: https://orcid.org/0000-0002-8562-1337

Universidade Federal de São João del Rei, Brasil E-mail: csanches@ufsj.edu.br

\begin{abstract}
Resumo
Ao longo dos anos, houve uma crescente busca para se ofertar um serviço de qualidade e com isso, inseriu-se os Núcleos de Segurança ao Paciente (NSP) nos hospitais, sendo uma estratégia para a melhoria contínua do serviço de saúde. O objetivo do estudo foi analisar a composição e as ações voltadas à segurança do paciente realizadas por NSP em hospitais brasileiros na perspetiva de profissionais de saúde que atuam nestas instâncias. Trata-se de um estudo realizado por meio de um questionário on-line. Selecionou-se hospitais de grande e médio porte registados no CNES e logo após buscou-se seus e-mails institucionais, convidando-os a participarem da pesquisa. Participaram do estudo 107 hospitais de todas as regiões do país, sendo a maior parte de natureza filantrópica $(46,9 \%)$ e complexidade média $(51,6 \%)$. Em $84,4 \%$, os NSP possuem representatividade dos membros da equipe, de forma integrada (71,9\%). Mas verificou-se uma deficiência quanto ao uso de indicadores e instrumentos de avaliação da cultura de segurança $(62,5 \%)$. Os resultados da pesquisa permitiram um panorama da situação em que se encontram alguns NSP no território brasileiro. Contudo, observa-se a necessidade de avanços e do apoio da liderança hospitalar, para um fortalecimento da cultura de segurança do paciente.
\end{abstract}

Palavras-chave: Segurança do paciente; Qualidade da assistência à saúde; Gestão de risco.

\begin{abstract}
There has been a growing search focusing on offering a service of high quality and one strategy for the continuous improvement of the health service are Patient Safety Centers (PSC). This study aimed to analyze the composition and actions to patient safety improvement carried out by PSC in Brazilian hospitals from the perspective of health professionals who work in these instances. This is a study carried out using an online questionnaire. Large and medium-sized hospitals were selected, and their institutional e-mails were sought, inviting them to participate in the research. 107 hospitals from all regions of the country participated in the study, most of them philanthropic (46.9\%) and medium complexity (51.6\%). In $84.4 \%$, PSCs have representativeness of team members, in an integrated manner $(71.9 \%)$. However, there was a deficiency in the use of indicators and instruments for assessing the safety culture $(62.5 \%)$. These results allowed an overview of the actual situation in which some PSCs are found in Brazilian territory. However, there is a need for improvement and the support of hospital leadership to strengthen the culture of patient safety.
\end{abstract}

Keywords: Patient safety; Quality of health care; Risk management. 


\section{Resumen}

A lo largo de los años, ha habido una búsqueda creciente por ofrecer un servicio de calidad y con ello, los Centros de Seguridad del Paciente (NSP) se han insertado en los hospitales, siendo una estrategia para la mejora continua del servicio de salud. El objetivo del estudio fue analizar la composición y las acciones dirigidas a la seguridad del paciente que realizan los NSP en los hospitales brasileños desde la perspectiva de los profesionales de la salud que actúan en estas instancias. Se trata de un estudio realizado mediante un cuestionario online. Se seleccionaron los hospitales grandes y medianos registrados en el CNES y, poco después, se buscaron sus correos electrónicos institucionales, invitándolos a participar en la investigación. En el estudio participaron 107 hospitales de todas las regiones del país, la mayoría filantrópicos $(46,9 \%)$ y de mediana complejidad (51,6\%). En el 84,4\%, los NSP tienen representatividad de los miembros del equipo, de manera integrada (71,9\%). Sin embargo, hubo una deficiencia en el uso de indicadores e instrumentos para la evaluación de la cultura de seguridad $(62,5 \%)$. Los resultados de la investigación permitieron tener una visión general de la situación en la que se encuentran algunos NSP en el territorio brasileño. Sin embargo, existe la necesidad de avances y apoyo del liderazgo hospitalario para fortalecer la cultura de seguridad del paciente.

Palabras clave: Seguridad del paciente; Calidad de la atención sanitaria; Gestión sanitaria rasguño.

\section{Introdução}

Em 1999, o relatório To err is human: building a safer health system publicado pelo Institute of Medicine (IOM), explorou uma importante dimensão da qualidade, que é a segurança do paciente. Este documento estimou que anualmente os eventos adversos são responsáveis por quase cem mil óbitos nos Estados Unidos da América (EUA) (Kohn, et al., 2000). No Brasil, um estudo pioneiro realizado em três hospitais de ensino do Rio de Janeiro avaliando 1.103 prontuários, mostrou que a incidência de pacientes com eventos adversos foi de 7,6\%, sendo que a proporção geral de eventos adversos evitáveis foi de 66,7\% e a densidade de incidência foi de 0,8 por 1.000 pacientes-dia (Mendes, et al., 2009).

Nestes cenários, nacional e internacional de falhas evitáveis na assistência à saúde, várias estratégias têm sido adotadas para prevenir, detectar e mitigar os eventos adversos, no intuito de reduzir danos preveníveis. No Brasil, em 2013 foi instituído o Programa Nacional de Segurança do Paciente (PNSP) (Brasil, 2013a), reforçado pela Resolução RDC no 36 de 2013, da Agência Nacional de Vigilância Sanitária (ANVISA) (Brasil, 2013b), que estabeleceu ações obrigatórias para a promoção da segurança do paciente nos serviços de saúde brasileiros, incluindo todos hospitais, públicos e privados. Dentre essas ações, determinou-se a criação de uma instância denominada Núcleo de Segurança do Paciente (NSP), que deve ser instituída pela direção do hospital (Brasil, 2013b).

O NSP deve ser composto por uma equipe multidisciplinar, capacitada quanto aos princípios da segurança do paciente. Por meio da elaboração e implantação de um Plano de Segurança do Paciente (PSP), o NSP realiza diversas ações para promoção da segurança do paciente como integrar as diferentes instâncias do hospital para o gerenciamento dos riscos e notificar os eventos adversos ao Sistema de Notificação de Eventos Adversos à Vigilância Sanitária (NOTIVISA) (Brasil, 2013c, Brasil, 2016).

A implantação dos NSP pelos serviços de saúde vem ocorrendo de forma gradativa desde a instituição do PNSP. Em 2014, em todo território nacional e nos diversos serviços de saúde havia apenas 784 NSP cadastrados na ANVISA (Brasil, 2015), já em 2019 o número aumentou para 4.549, incluindo NSP instituídos nos hospitais (Brasil, 2019). Destes, cerca de 44,2\% localizam-se na região Sudeste; $19,2 \%$ na região Sul; 17,1\% na região Nordeste; $14,9 \%$ na região Centro-Oeste e 4,6\% na região Norte (Brasil, 2019). A notificação de incidentes no NOTIVISA é uma importante atribuição dos NSP e tem aumentado significativamente ao longo dos anos (Brasil, 2013b, Brasil, 2019). De 2014 à 2019 foram 330.536 incidentes notificados, sendo a maior parte deles realizados por hospitais $(93,4 \%)$ e destes $44,1 \%$ foram da região Sudeste, seguido da região Sul (20,1\%), Nordeste (18,7\%), Centro-Oeste (12,8\%) e Norte (4,3\%) (Brasil, 2019).

Nota-se significativos avanços na promoção da segurança do paciente, na implantação dos NSP e notificação de incidentes por estas instâncias à ANVISA (Prates, et al., 2019). No entanto, a constituição de um NSP e a implantação de ações para a segurança do paciente em hospitais brasileiros ainda é complexo e desafiador, havendo desde limitações relacionadas à 
recursos financeiros, até uma frágil cultura de segurança do paciente. Além de um desconhecimento pelos profissionais de saúde em como implantar e desenvolver as ações de um NSP (Prates, et al., 2019, Cavalcante, et al., 2019).

Estudos presentes na literatura evidenciam a importância e também os benefícios de um NSP atuante nos serviços de saúde (Prates, et al., 2019, Santos, et al., 2019). Nesse contexto, o objetivo deste estudo é analisar a composição e as ações voltadas à segurança do paciente realizadas por Núcleos de Segurança do Paciente em hospitais brasileiros na perspectiva de profissionais de saúde que atuam nestas instâncias.

\section{Metodologia}

\subsection{Desenho do estudo}

Estudo transversal, quantitativo, descritivo, que utilizou dados sobre os NSP de hospitais de médio e grande porte, de todas as Unidades da Federação brasileira, registrados no Cadastro Nacional de Estabelecimentos de Saúde (CNES), disponibilizados pelo Departamento de Informática do Sistema Único de Saúde (DATASUS). Segundo a legislação nacional, é considerado hospital de médio porte aquele com 51 a 150 leitos e hospital de grande porte aquele com 151 a 500 leitos (Negri, et al., 2014, Brasil, 1977). Os hospitais ao se registrarem no CNES fornecem esta informação e classificam seu porte.

\subsection{População e amostra}

Localizou-se 6.385 hospitais de médio e grande porte cadastrados no CNES. Destes, 687 não disponibilizavam endereço eletrônico para consulta e 908 continham cadastro duplicado na plataforma. Totalizando portanto 4.790 e-mails de hospitais, elegíveis à participarem do estudo. Conduziu-se o envio dos e-mails e 2.524 retornaram com alerta de erro. Para estes, procedeu-se à uma nova coleta de endereços eletrônicos no site oficial de cada hospital, e mais 1.533 endereços eletrônicos foram adquiridos, totalizando 3.799 e-mails. Destes, 1.581 hospitais retornaram novamente com problemas de recebimento no e-mail. Portanto, contabilizou-se uma amostragem final de 2.218 e-mails entregues aos hospitais.

\subsection{Instrumento de coleta de dados}

Trata-se de um estudo realizado por meio de um questionário eletrônico enviado via e-mail. O referido e-mail enviado continha uma solicitação de redirecionamento a um membro do NSP, convidando-o a participar da pesquisa. Forneceu-se um link para acesso ao questionário eletrônico via plataforma GoogleDocs, o qual era acessado após o aceite em participar do estudo mediante Termo de Consentimento Livre e Esclarecido (TCLE).

O questionário eletrônico foi construído em duas seções. A primeira com questões sociodemográficas e econômicas do hospital, como a composição do NSP e o nível de complexidade do hospital. A segunda seção foi elaborada pelos pesquisadores a partir das legislações e documentos do Ministério da Saúde e da ANVISA sobre NSP (Brasil, 2013a, Brasil, 2013b, Brasil, 2013c). Assim, contendo questões sobre as competências do NSP, ações de promoção da segurança do paciente no hospital, condições organizacionais, processos de trabalho e notificação dos eventos adversos ao NOTIVISA. As questões eram de múltipla escolha e os membros do NSP poderiam responder "Sim", "Não", "Em implantação", "Parcialmente" e "Não sei/não se aplica”.

\subsection{Período de coleta dos dados}

A coleta de dados ocorreu de janeiro a julho de 2019. Este período foi uma estratégia para obter maior adesão dos profissionais que atuam nos NSP, dada a abrangência nacional e as dificuldades das pesquisas que utilizam questionários eletrônicos, as quais tem adesão variável (Andrade, et al., 2018, Braithwaite, et al., 2003, Saced, et al., 2005). Dos 2.218 emails obtidos via CNES e páginas eletrônicas oficiais dos hospitais, enviou-se três lembretes para cada instituição, no prazo de 
1,2 e 3 meses após a primeira tentativa de contato.

\subsection{Tratamento e análise dos dados}

Por fim, realizou-se a análise estatística descritiva por meio de distribuição de frequência absoluta e relativa. O tratamento estatístico dos resultados obtidos foi realizado por meio da aplicação do software SPSS 19.0 (IBM SPSS Statistics $\left.{ }^{\mathrm{TM}}\right)$. A pesquisa foi aprovada pelo Comitê de Ética em Pesquisa Envolvendo Seres Humanos do Campus Centro Oeste Dona Lindu da Universidade Federal de São João del-Rei, sob parecer nº 2.940.875 e CAAE nº 98680718.8.0000.5545.

\section{Resultados}

Após o envio do questionário, 2.110 hospitais não responderam à pesquisa, 01 hospital se recusou e 107 hospitais aceitaram a participar da pesquisa. Sendo assim, a taxa de resposta desta pesquisa foi de 4,82\%. Dos 107 hospitais participantes, 43 preencheram de forma incompleta uma ou as duas seções do questionário eletrônico. Assim, participaram da pesquisa 64 hospitais, a maioria são filantrópicos $(46,9 \%)$ e de média complexidade (51,6\%). Os hospitais de médio porte corresponderam a $57,8 \%$ e os de grande porte a $42,2 \%$ dos hospitais (Tabela 1 ).

Tabela 1: Perfil dos hospitais participantes da pesquisa, Brasil (2019), ( $\mathrm{n}=64)$.

\begin{tabular}{lcc}
\hline Variáveis & N & $\%$ \\
\hline Região & & \\
Sudeste (\%) & 12 & 18,9 \\
Minas Gerais & 9 & 14,2 \\
São Paulo & 3 & 4,6 \\
Espírito Santo & 2 & 3,1 \\
Rio de Janeiro & & \\
Sul (\%) & 8 & 12,6 \\
Paraná & 6 & 9,4 \\
Santa Catarina & 6 & 9,4 \\
Rio Grande do Sul & & \\
Nordeste (\%) & 4 & 6,3 \\
Pernambuco & 3 & 4,6 \\
Bahia & 1 & 1,5 \\
Maranhão & 1 & 1,5 \\
Alagoas & & \\
Centro-Oeste (\%) & & 3,1 \\
Mato Grosso do Sul & 3 & \\
Goiás & 2 & \\
Norte (\%) & & \\
Pará & & \\
\hline & & \\
\hline
\end{tabular}




\begin{tabular}{|c|c|c|}
\hline Tocantins & 2 & 3,1 \\
\hline \multicolumn{3}{|l|}{ Natureza do hospital (\%) } \\
\hline Filantrópico & 30 & 46,9 \\
\hline Público & 23 & 35,9 \\
\hline Privado & 11 & 17,2 \\
\hline \multicolumn{3}{|c|}{ Nível de complexidade do hospital (\%) } \\
\hline Médio & 33 & 51,6 \\
\hline Alto & 25 & 39,1 \\
\hline Não sei / Não se aplica & 6 & 9,3 \\
\hline Número de leitos (média) & 143 & - \\
\hline \multicolumn{3}{|c|}{ Tempo de funcionamento do NSP $(\%)$} \\
\hline$\geq 4$ anos & Conclusão 41 & 64,1 \\
\hline$\leq 1$ ano & 12 & $\begin{array}{c}\text { Continua } \\
\qquad,,\end{array}$ \\
\hline 3 anos & 11 & 17,2 \\
\hline \multicolumn{3}{|c|}{ Período de reuniões do NSP (\%) } \\
\hline Mensalmente & 38 & 59,4 \\
\hline Não sei & 10 & 15,6 \\
\hline Bimestralmente & 8 & 12,5 \\
\hline Quinzenalmente & 6 & 9,4 \\
\hline Semanalmente & 2 & 3,1 \\
\hline
\end{tabular}

Fonte: Autores.

Participaram do estudo hospitais de todas as regiões do país, sendo a maior parte da região Sudeste (40,8\%) e o menor número foi da região Norte $(6,2 \%)$. Houve ausência de representatividade de hospitais de alguns estados. Na região CentroOeste não obteve-se participação de hospitais do estado do Mato Grosso e nas regiões Norte e Nordeste essa ausência foi de 5 estados cada região, sendo eles: os estados do Amazonas, Roraima, Amapá, Rondônia e Acre (região Norte) e os estados do Piauí, Ceará, Rio Grande do Norte, Paraíba e Sergipe (região Nordeste). As regiões Sudeste e Sul tiveram participação de no mínimo dois hospitais de cada um de seus estados (Tabela 1).

Todos os hospitais que participaram da pesquisa possuem um NSP, a maioria (42,2\%) estão implantados há mais de quatro anos e realizam reuniões mensais $(59,4 \%)$, segundo os participantes (Tabela 1). Os NSP participantes estão compostos por equipe multiprofissional, em 93,7\% destas instâncias há a participação de enfermeiros, seguido de farmacêuticos (84,4\%), médicos $(65,6 \%)$ e nutricionistas $(26,6 \%)$.

Segundo os participantes, a direção nomeou e realizou a composição da maior parte dos NSP (65,6\%). No entanto, apenas $39,1 \%$ da liderança destes hospitais fornecem adequadamente recursos humanos e financeiros; equipamentos, insumos e materiais, impactando negativamente o funcionamento dos NSP. Em contrapartida, os participantes em sua maioria (53,1\%) declararam que há garantias das boas práticas de funcionamento do NSP e que a conformação do NSP é adequada ao tipo e a complexidade do serviço $(60,9 \%)$ (Tabela 2$)$. 
Tabela 2: Processos estabelecidos e taxa de afirmações quanto às práticas institucionais dos Núcleos de Segurança do Paciente em hospitais brasileiros, (2019), $(n=64)$.

\begin{tabular}{|c|c|c|c|}
\hline \multirow{2}{*}{ Variáveis } & \multicolumn{3}{|c|}{$\%$} \\
\hline & Sim & Não & Parcialmente \\
\hline $\begin{array}{l}\text { O NSP possui representantes das áreas de controle de infecção, qualidade } \\
\text { farmácia hospitalar, serviço de enfermagem, dentre outros? }\end{array}$ & 65,6 & 7,8 & 26,6 \\
\hline $\begin{array}{l}\text { A direção fornece recursos humanos, financeiros, equipamentos, insumos e } \\
\text { materiais para o adequado funcionamento do NSP? }\end{array}$ & 39,1 & 12,5 & 48,4 \\
\hline $\begin{array}{l}\text { Há garantia das boas práticas de funcionamento do NSP no serviço de saúde } \\
\text { dentro de seu âmbito de atuação? }\end{array}$ & 53,1 & 7,8 & 39,2 \\
\hline A conformação do NSP é adequada ao tipo e a complexidade do serviço? & 60,9 & 12,5 & 26,6 \\
\hline $\begin{array}{l}\text { A equipe é capacitada em conceitos de melhoria da qualidade, segurança do } \\
\text { paciente e em ferramentas de gerenciamento de riscos em serviços de } \\
\text { saúde? }\end{array}$ & 51,6 & 7,8 & 40,6 \\
\hline $\begin{array}{l}\text { Ocorre integração das diferentes instâncias que trabalham com riscos na } \\
\text { instituição? }\end{array}$ & 71,9 & 7,3 & 20,3 \\
\hline $\begin{array}{l}\text { Há promoção de trabalho em equipe com um enfoque proativo, sistemático } \\
\text { e organizacional de trabalho? }\end{array}$ & 51,6 & 6,2 & 42,2 \\
\hline $\begin{array}{l}\text { Seus membros possuem autoridade, responsabilidade e poder para executar } \\
\text { as ações do Plano de Segurança do Paciente (PSP)? }\end{array}$ & 57,8 & 6,3 & 35,9 \\
\hline $\begin{array}{l}\text { A elaboração do PSP teve participação de todas as lideranças dos setores da } \\
\text { instituição? }\end{array}$ & 42,2 & 21,9 & 35,9 \\
\hline $\begin{array}{l}\text { O NSP possui um cronograma de atividades e tarefas estabelecidas para } \\
\text { melhoria da segurança, com os responsáveis pelas ações e documentação } \\
\text { dos progressos obtidos? }\end{array}$ & 46,9 & 12,5 & 40,6 \\
\hline $\begin{array}{l}\text { O NSP faz uso de indicadores, que permitem representar de forma } \\
\text { quantitativa, a evolução e o desempenho de um determinado processo, da } \\
\text { qualidade dos produtos e serviços, da participação ou da motivação de } \\
\text { colaboradores de uma instituição? }\end{array}$ & 50,0 & 18,8 & 31,2 \\
\hline $\begin{array}{l}\text { Há melhoria contínua dos processos de cuidado e do uso de tecnologias da } \\
\text { saúde?" }\end{array}$ & 51,6 & 6,2 & 42,2 \\
\hline Na sua instituição o paciente tem liberdade para participar do NSP? & 7,8 & 64,1 & 28,1 \\
\hline
\end{tabular}


Há mecanismos de retornar ao paciente os resultados da assistência à saúde? $\quad 26,6 \quad 37,5 \quad 35,9$

São usados instrumentos de avaliação da cultura de segurança, como o

15,6

62,5

21,9 Hospital "Survey on Patient Safety Culture" (HSOPSC) e "Safety Attitudes Questionnaire" (SAQ)?

Fonte: Autores.

A capacitação dos membros dos NSP, tão necessária aos princípios de melhoria da qualidade, segurança do paciente e ferramentas de gerenciamento de riscos está presente em 51,6\% dos NSP, segundo os participantes. Na grande maioria dos NSP participantes, há representação de profissionais vinculados às áreas de controle de infecção, qualidade e farmácia hospitalar (84,4\%). Essa afirmativa colabora na integração das diferentes instâncias que trabalham com riscos dentro dos hospitais, citada por $71,9 \%$ dos participantes da pesquisa e que há promoção de trabalho em equipe em aproximadamente $51,6 \%$ dos hospitais (Tabela 2).

O PSP é uma atribuição inicial de qualquer NSP, de acordo com a maioria dos participantes $(57,8 \%)$ eles possuem autoridade, responsabilidade e poder para executar as ações propostas neste documento. Porém, isso ocorre de forma parcial na realidade de 35,9\% dos NSP e não ocorre em 6,3\%, prejudicando a promoção das ações de segurança do paciente nos hospitais. Além disso, de acordo com os respondentes, a elaboração deste documento teve participação das lideranças da instituição em menos da metade dos hospitais $(42,2 \%)$ e em 35,9\% esta participação ocorreu de forma parcial.

É importante que os NSP possuam um cronograma de atividades e tarefas estabelecidas para melhoria da segurança, contemplando os responsáveis pelas ações os progressos obtidos. Este planejamento e acompanhamento é observado em apenas 46,9\% dos hospitais e em 40,6\% os respondentes declararam que isto ocorre de forma parcial. O uso de indicadores também é fundamental para visualizar a evolução de um determinado processo, acompanhar a qualidade dos produtos e serviços e a participação e motivação de colaboradores do hospital. Metade dos participantes da pesquisa afirmam possuir esses indicadores e 31,2\% responderam a alternativa parcialmente sobre esta questão. Mesmo neste contexto de dificuldade de gerenciamento dos dados institucionais, apenas $6,2 \%$ dos profissionais afirmam que não há melhoria contínua dos processos de cuidado e do uso de tecnologias da saúde (Tabela 2).

A participação de pacientes nos NSP foi positiva em apenas 7,8\%, segundos os membros participantes do estudo. Entretanto, mais da metade dos profissionais relataram que há mecanismos de retornar ao paciente os resultados da assistência do hospital, sendo que 35,9\% responderam que é de forma parcial. Ademais, a maioria (62,5\%) dos hospitais não realizam avaliação de cultura da segurança com o uso de instrumentos como o "Hospital Survey on Patient Safety Culture" (HSOPSC) e "Safety Attitudes Questionnaire" (SAQ) (Tabela 2).

Uma importante atribuição de qualquer NSP é a detecção precoce e mitigação de incidentes e eventos adversos, isso tem ocorrido em 56,2\% dos NSP cenários do estudo e em grande parcela apenas de forma parcial (31,3\%). Contraditoriamente, $75,0 \%$ dos participantes relatam que há barreiras para a prevenção de incidentes (Tabela 3). 
Tabela 3: Interação do Núcleo de Segurança do Paciente com a Vigilância Sanitária em hospitais brasileiros, (2019), (n= 64).

\begin{tabular}{|c|c|c|c|c|}
\hline \multirow{2}{*}{ Variáveis } & \multicolumn{4}{|c|}{$\%$} \\
\hline & Sim & Não & Parcialmente & $\begin{array}{c}\text { Não sei / } \\
\text { Não se aplica }\end{array}$ \\
\hline O NSP trabalha na detecção precoce e mitigação de eventos adversos? & 56,2 & 12,5 & 31,3 & - \\
\hline $\begin{array}{l}\text { Há barreiras de segurança para a prevenção de incidentes em seu } \\
\text { hospital? }\end{array}$ & 75,0 & 10,9 & 14,1 & - \\
\hline $\begin{array}{l}\text { Além da notificação pelos profissionais, o NSP tem outra fonte de } \\
\text { monitoramento e avaliação para captar os dados sobre incidentes, como } \\
\text { busca ativa em prontuários, walkrounds e auditoria da qualidade? }\end{array}$ & 37,5 & 29,7 & 23,4 & 9,4 \\
\hline Ocorre fiscalização da vigilância sanitária local? & 90,6 & 3,1 & 6,3 & - \\
\hline $\begin{array}{l}\text { O Coordenador do NSP é o principal contato da instituição com a equipe } \\
\text { do Sistema Nacional de Vigilância Sanitária? }\end{array}$ & 53,1 & 18,8 & 15,6 & 12,5 \\
\hline $\begin{array}{l}\text { O registro de incidentes é realizado no módulo específico do Sistema de } \\
\text { Notificações em Vigilância Sanitária (NOTIVISA)? }\end{array}$ & 57,8 & 12,5 & 18,8 & 10,9 \\
\hline $\begin{array}{l}\text { O NSP tem conseguido realizar a notificação dos eventos adversos } \\
\text { mensalmente até o } 15^{\circ} \text { dia útil do mês subsequente ao mês de vigilância } \\
\text { à ANVISA? }\end{array}$ & 39,1 & 28,1 & 32,8 & - \\
\hline $\begin{array}{l}\text { O NSP tem conseguido realizar a notificação dos eventos adversos que } \\
\text { evoluíram para óbito em até } 72 \text { horas a partir do ocorrido? }\end{array}$ & 50,0 & 29,7 & 20,3 & - \\
\hline
\end{tabular}

Fonte: Autores.

Em um local de aprendizado coletivo, os profissionais são estimulados a notificar os incidentes relacionados à assistência à saúde sem ameaça e punição, criando um ambiente onde riscos, falhas e danos podem ser facilmente reportados 5 . Esta é uma realidade de 68,7\% segundo os respondentes da pesquisa. Sendo que em 65,7\% é atribuição dos NSP a notificação de eventos adversos ao Sistema Nacional de Vigilância Sanitária (SNVS). Ademais, 37,5\% dos participantes afirmaram que além da notificação pelos profissionais, o NSP tem outras fontes de monitoramento e avaliação para captar os dados sobre incidentes, como busca a ativa em prontuários, walkrounds e auditoria da qualidade (Tabela 3).

A grande maioria dos respondentes da pesquisa $(90,6 \%)$ afirmaram que há fiscalização da vigilância sanitária local. Porém, pouco mais da metade $(53,1 \%)$ alegaram que o coordenador do NSP é o principal contato da instituição com a equipe do SNVS e apenas 57,8\% mencionaram que o registro de incidentes é realizado no NOTIVISA. Chama a atenção, que $10,9 \%$ dos participantes relataram que não sabem ou não se aplica a notificação pelo NOTIVISA (Tabela 3).

A notificação dos eventos adversos pelo NSP é obrigatória pela legislação, até o $15^{\circ}$ (décimo quinto) dia útil do mês subsequente ao mês que ocorreu o evento (Brasil, 2013b). Apenas 39,1\% dos NSP relataram conseguir realizar esta ação e 32,8\% conseguem de forma parcial. Contudo, a metade dos NSP tem conseguido realizar a notificação no NOTIVISA dos eventos adversos que evoluíram para óbito em até 72 (setenta e duas) horas a partir do ocorrido (Tabela 3). 


\section{Discussão}

O perfil de complexidade e natureza jurídica dos hospitais participantes da pesquisa foi em sua maioria de média complexidade e filantrópicos, semelhantemente ao perfil encontrado em outro estudo brasileiro que também analisou hospitais de médio e grande porte (Santos, et al., 2018).

O sudeste e norte foram as regiões com maior e menor número de hospitais participantes do estudo, respectivamente. Podendo estar relacionado a serem as regiões com maior e menor número total de estabelecimentos de saúde que possuem leitos no Brasil (Brasil, 2017). Dos 11 estados que não tiveram participação de nenhum hospital neste estudo, quatro (Sergipe, Ceará, Amapá, Mato Grosso) também não preencheram o cadastramento junto à ANVISA, que tem uma Coordenação Estadual dos Núcleos de Segurança do Paciente em 2019 (Brasil, 2019), mostrando falta de estímulo destas Secretarias Estaduais de Saúde no estímulo de implantação e promoção da segurança do paciente pelos serviços de saúde. Por outro lado, as regiões sudeste e sul que tiveram participação de hospitais de todos os seus estados, também se destacam nas ações e estudos sobre segurança do paciente (Macedo \& Boliomol, 2019, Siman, et al., 2017,Bao, et al., 2019,Sartor, et al., 2016,Rennó, et al., 2018).

A totalidade dos participantes da pesquisa afirmaram a existência de um NSP em seu hospital, a maioria implantado a mais de quatro anos e com reuniões mensais. As reuniões são cruciais para a discussão de ações necessárias e compartilhamento de progressos e resultados obtidos, sendo interessante convidar a gerência e representantes de instâncias que trabalham com riscos à participarem das reuniões para contribuírem e se inteirarem das demandas. Cada instância deve estabelecer o período de suas reuniões, assim como as pautas a serem discutidas, e as mesmas devem ser devidamente documentadas 5. Os participantes da pesquisa afirmaram que os NSP são compostos por equipe multiprofissional. No entanto, não são todos os NSP que tem a composição designado pela legislação, a qual orienta que os NSP devem ser minimamente composta por enfermeiros, farmacêuticos e médicos (Brasil, 2013b).

A direção dos hospitais nomeou e a definiu a composição da maior parte dos NSP cenário do estudo, conforme legislação vigente (Brasil, 2013b). Porém, o fornecimento de recursos humanos e financeiros, equipamentos, insumos e materiais pela direção, não tem sido realidade em todos os hospitais. Neste contexto, a percepção positiva dos membros dos NSP participantes do estudo quanto à adequada conformação e a garantia das boas práticas de funcionamento desta instância, torna-se questionável.

Um estudo realizado no nordeste brasileiro demonstrou que recursos financeiros são apresentados como uma das dificuldades para a formação do NSP e desenvolvimento de suas ações (Azevedo, et al., 2016). Outro estudo também na região nordeste, dos (Macedo \& Boliomol, 2019) NSP avaliados, apenas quatro (33,3\%) dispõem de recursos financeiros próprios para o desenvolvimento das atividades de segurança (Costa, et al., 2020). Sem os recursos básicos exigidos pela legislação, o trabalho de promoção da segurança do paciente fica comprometido. Além disso, é o comportamento e o comprometimento da gestão hospitalar com o NSP que incentiva os membros e os demais profissionais a realizarem mudanças necessárias para o alcance da melhoria contínua da segurança do paciente (Cavalcante, et al., 2019, McFadden, et al., 2015).

De acordo com os dados obtidos, pouco mais da metade dos membros dos NSP participantes são capacitados em princípios de melhoria da qualidade, segurança do paciente e ferramentas de gerenciamento de riscos. O processo de capacitação dos NSP ainda está em desenvolvimento e é uma realidade em outros hospitais do Brasil (Macedo \& Boliomol, 2019), possivelmente explicado pelo pouco tempo de atuação dos NSP de um modo geral.

Uma pesquisa realizada em unidades de emergência de dois hospitais da Suécia, revelou que a implementação de um projeto de melhoria da qualidade resultou em evolução do trabalho, maior abertura para comunicação e melhor trabalho em equipe entre unidades dos hospitais (Burstrom, et al., 2014). Assim, é necessário a proposição de programas contínuos de capacitação e educação permanente como ferramentas para mudanças no processo de trabalho dos profissionais (Cavalcante, et al., 2019, Macedo \& Boliomol, 2019). 
A participação de profissionais das áreas de controle de infecção, qualidade e farmácia hospitalar ocorre na maioria dos NSP do estudo, porém ainda é necessário maior integração destas áreas para o gerenciamento dos riscos dentro dos hospitais e que o trabalho em equipe seja fortalecido. Corroborando a estas informações, um estudo realizado em hospitais sentinela de São Paulo também mostrou como a integração dos diferentes processos de gestão de risco desenvolvidos no serviço é desafiador, apenas um hospital afirmou conseguir realizar de forma total, sendo que a grande maioria o faz de forma parcial (Macedo \& Boliomol, 2019). Porém, é imprescindível que o NSP contenha representantes e profissionais das diferentes áreas dentro da instituição, sendo que o trabalho em equipe reflete grande impacto na segurança do paciente, sendo considerado um fator determinante para a prevenção de falhas e eventos adversos (Brasil, 2013c,Andrade, et al., 2018, Abu-ElNoor, et al., 2019).

O trabalho da equipe multiprofissional do hospital para promoção da segurança do paciente deve ser discutido entre todos e constar no PSP elaborado pelos NSP. Um estudo realizado em hospitais no estado São Paulo, mostrou a importância dos integrantes dos NSP possuírem autoridade para executar o PSP e do apoio da direção (Macedo \& Boliomol, 2019). Porém, quase metade dos participantes do estudo afirmaram não possuir autoridade, responsabilidade e poder para executar as ações do PSP. Isso pode ser explicado, dentre outros fatores, pelo fato de pouco mais da metade dos hospitais do estudo contarem com a participação das lideranças no processo de construção deste importante documento, contrariando a legislação vigente (Brasil, 2013b).

Isso também será importante para elaboração de um cronograma das atividades, o qual deve possuir os responsáveis por cada ação e o registro obtido das melhorias em segurança (Brasil, 2013c). Segundo os participantes, há este acompanhamento em menos da metade dos hospitais e em muitos ocorre de forma parcial, o que prejudica o gerenciamento das ações. $\mathrm{O}$ uso de indicadores pelos NSP participantes também precisa expandir para a totalidade dos hospitais. Os indicadores são indispensáveis para o gerenciamento de boas práticas no ambiente hospitalar (Rossaneis, et al., 2015). Porém, seu uso ainda é prejudicado pela dificuldade na obtenção dos dados para análise e falta de treinamento dos profissionais, o que compromete sua utilização e compreensão do indicador (Rossaneis, et al., 2015,Lima, et al., 2015).

Chama a atenção que ao mesmo tempo que os membros dos NSP participantes do estudo, relatam não terem um gerenciamento efetivo das ações e dos dados por meio de cronograma de atividade e indicadores de melhoria, apenas quatro NSP afirmam que não há melhoria contínua dos processos de cuidado e do uso de tecnologias da saúde. Assim, infere-se que a grande maioria afirmaram possuir melhorias sem possuírem informações fidedignas (Macedo \& Boliomol, 2019,Becker, et al., 2018).

Apesar da grande maioria dos profissionais participantes alegarem não ter participação de pacientes nos NSP, mais da metade relataram que há mecanismos de retornar ao paciente os resultados da assistência do hospital. Semelhantemente, em um estudo brasileiro com coordenadores de NSP, o incentivo para que o paciente e seus familiares se envolverem em sua própria segurança estava no planejamento de apenas metade dos hospitais (Macedo \& Boliomol, 2019). A falta de oportunidade promovida pelos hospitais para participação efetiva do paciente e familiares nas ações de segurança, leva a estes uma falta de conhecimento sobre seus direitos e conceitos de segurança do paciente. Isso foi demonstrado em um estudo nacional (Macedo \& Boliomol, 2019) e também em uma pesquisa realizada no Reino Unido (Ricci-Cabello, et al., 2016). Porém, é fundamental que o paciente esteja envolvido em seu próprio processo do cuidado, sendo necessário sensibilizar os profissionais sobre o desenvolvimento desta ação para promover um cuidado seguro (Cavalcante, et al., 2019,Ricci-Cabello, et al., 2016,Smith, et al., 2017).

A avaliação da cultura de segurança não é realizada pela maioria dos NSP participantes. A cultura de segurança é o conjunto de valores, atitudes, competências e comportamentos que produzem o comprometimento com a gestão da saúde e da segurança e substituiu a culpa e a punição pela oportunidade de aprender com as falhas, e melhorar a atenção à saúde (Brasil, 
2013b). A avaliação da cultura de segurança é uma ferramenta que permite o reconhecimento da situação hospitalar, identificando problemas de segurança nas rotinas e condições de trabalho para gerenciá-los prospectivamente (Santiago \& Turrini, 2015, Reis, et al., 2018).

Um estudo de revisão da literatura identificou diferentes instrumentos para a avaliação da cultura de segurança, sendo que o Safety Attitudes Questionnaire (SAQ) e o Hospital Survey on Patient Safety Culture (HSOPSC) são muito utilizados em hospitais, bem como o Patient Safety Culture in Healthcare Organizations Survey (PSCHO) e o Modified Stanford Patient Safety Culture Survey Instrument (MSI) (Halligari, et al., 2014). Os membros dos NSP devem analisar os instrumentos disponíveis e decidir qual deles estão mais adequados a sua realidade e aplicá-los de modo que os resultados norteiem seu planejamento para a promoção da segurança do paciente.

Ações para detectar os incidentes e eventos adversos antes que atinjam os pacientes tem ocorrido nos NSP do estudo, segundo os participantes. No entanto, uma grande parcela ocorre apenas de forma parcial e mesmo assim, a maioria dos participantes relatam que há barreiras para a prevenção de incidentes. Essas declarações se contradizem, uma vez que se deve haver um sinergismo entre a detecção precoce dos eventos adversos e a implantação de barreiras. Os NSP devem conhecer o processo de cuidado em seu serviço, para que consigam identificar pontos críticos e trabalhar na prevenção, deteç̧ão precoce e mitigação de erros (Brasil, 2013c). A gestão de riscos é entendida como a aplicação de políticas de segurança e estratégias de ações, que contemplam desde à identificação até o controle dos riscos e eventos adversos que possam atingir o paciente, o profissional, o meio ambiente e a instituição. Isso culmina no estabelecimento de barreiras para prevenção de incidentes, sendo o pilar de qualquer instituição que busca melhoria nos cuidados em saúde (Brasil, 2013b, McFadden, et al., 2015).

Na perspectiva da maior parte dos participantes da pesquisa, os profissionais em seus hospitais possuem liberdade de notificar os incidentes relacionados à assistência à saúde sem ameaça e punição. Um estudo realizado em Salvador, consta que a maioria dos NSP estudados também estimulavam a notificação de incidentes pelos colaboradores do hospital (Costa, et al., 2020). No entanto, na maioria dos estudos que tratam sobre a notificação de incidentes, este ainda é um grande desafio dos hospitais. Outro estudo realizado no Rio Grande do Norte sobre a avaliação da cultura de segurança, mostrou que duas das principais dimensões frágeis foram em relação à notificação, quais sejam a dimensão de frequência de eventos notificados e de resposta não punitiva para erros (Andrade, et al., 2018).

Em um estudo brasileiro com 12 hospitais, apenas metade incentivam a notificação de eventos adversos sem a responsabilização individual (Macedo \& Boliomol, 2019). A subnotificação dos incidentes e eventos adversos são muitas vezes atreladas à sobrecarga de atividades, desconhecimento do processo de notificação, e principalmente à cultura negativa de notificações devido ao temor à punição (Siman, et al., 2017, Costa, et al., 2020). Assim, a conduta dos profissionais em relação ao erro pode variar de acordo com a cultura institucional (Siman, et al., 2017). Quando os profissionais se encontram em um ambiente de cultura organizacional positiva, que os encoraja no compartilhamento de lições aprendidas com os erros e promove melhoria de processos, o seu compromisso em notificar os erros aumenta substancialmente (Lemos, et al., 2018).

É atribuição da maioria dos NSP a notificação de eventos adversos ao SNVS. Alguns NSP alegam terem outras fontes de monitoramento e avaliação para captar os dados sobre incidentes, como busca ativa em prontuários, walkrounds e auditoria da qualidade. Cabe ao NSP monitorar os incidentes assim como notificar ao SNVS os eventos adversos decorrentes da prestação do serviço de saúde (Brasil, 2013b). A notificação dos erros e incidentes são reconhecidos como um elemento norteador de um programa de segurança em saúde, favorecendo uma cultura organizacional positiva e uma assistência de qualidade, uma vez que o conhecimento dos erros propicia a delimitação da grandeza desses eventos, cria oportunidades de melhorias, elaboração de indicadores, estruturação de barreiras de prevenção e tomada decisão (Costa, et al., 2020,Lemos, et al., 2018).

Outra variável importante é a fiscalização pela vigilância sanitária local, na qual, está sendo realizada em quase todos 
os hospitais participantes da pesquisa, uma vez que o funcionamento do NSP possui caráter compulsório, cabendo a este órgão a avaliação periódica (Brasil, 2013b). Chama a atenção que somente pouco mais da metade alegaram que o coordenador do NSP é o principal contato da instituição com a equipe do SNVS e que o registro de incidentes é realizado no NOTIVISA. Ressalta-se que alguns dos participantes membros do NSP, relataram que não sabem ou não se aplica a notificação pelo NOTIVISA. Muitos profissionais nomeados a coordenadores não desempenham atividades exclusivas do NSP, acumulando tarefas de funções simultâneas (Macedo \& Boliomol, 2019), podendo justificar os dados encontrados na pesquisa.

Identificou-se que nem todos NSP conseguem realizar a notificação ao SNVS dos eventos adversos ocorridos no hospital até o $15^{\circ}$ dia útil do mês subsequente e em até 72 horas os eventos adversos que evoluíram para óbito, ou a realizam de forma parcial. Assim, os eventos mais graves ocorridos na instituição não são comunicados a ANVISA em tempo real, isso também ocorreu em outro estudo brasileiro (Costa, et al., 2020). Este cenário contradiz o proposto pelo PNSP, em que o intuito deste prazo de notificação à ANVISA é para que este órgão acompanhe as ações propostas para a prevenção de erros semelhantes na instituição e contribua para a promoção de uma cultura de investigação (Brasil, 2013c,Siman, et al., 2017, Costa, et al., 2020).

Vale ponderar que apesar das contribuições, reconhecem-se algumas limitações na presente pesquisa. A primeira delas é o tamanho da amostra, a qual foi reduzida de forma significativa devido à incompletude das informações do CNES (Rocha, et al., 2018, Pelissari, 2019). No entanto, apesar dos resultados da pesquisa não poder ser generalizado para todos os NSP hospitalares existentes no Brasil, os 64 NSP participantes da pesquisa atenderam às expectativas de se obter um panorama de forma representativa da situação de alguns NSP implantados nas diferentes regiões do território nacional. Outra limitação é taxa de resposta de apenas 4,82\% dos hospitais elegíveis. Porém, destes elegíveis não se conhece quais são os hospitais tinham ou não um NSP implantado no momento da coleta de dados, sendo a não implantação do NSP um fator que pode ter colaborado para a taxa de respondentes. No entanto, apesar disso constituir de fato uma limitação, é também um ensejo para estudos futuros de seguimento, que inclusive está sendo buscado pelos pesquisadores.

\section{Conclusão}

Os resultados da pesquisa permitiram um panorama de forma representativa e preliminar da situação em que se encontram alguns NSP no território brasileiro. Identificou-se a presença de profissionais que representam as diferentes instâncias que trabalham com riscos atuando de maneira integrada, o que contribui para a visualização de fragilidades que demandam ações e estabelecimento de barreiras de segurança. Contudo, é extremamente necessário conferir autonomia a esses profissionais para execução de ações do PSP.

Foi possível constatar um déficit na maioria dos hospitais quanto ao gerenciamento de ações e dados e no uso de indicadores de melhoria, não permitindo o acompanhamento dos progressos. Verificou-se também, a necessidade de desenvolver estratégias para o envolvimento do paciente em seu próprio processo de cuidado, uma vez que este é o principal interessado no tema segurança.

Em relação a notificação de incidentes, este ainda é um desafio institucional, para que os hospitais e seus profissionais aprendam com os seus erros, os analisem e implantem processos de melhoria, entretanto nota-se avanços significativos neste seguimento. Assim, é importante que a liderança apoie e invista no fortalecimento da cultura de segurança, com ênfase na educação e comunicação dos profissionais, para que a prática de notificações se torne uma realidade em todos os hospitais. Além disso, os NSP precisam estabelecer fontes de monitoramento e avaliação de incidentes, para que junto com as notificações, essas informações sejam uma poderosa ferramenta de transformação. Sugere-se, como trabalhos futuros, a realização de estudos de campo, voltados para a avaliação da atuação, in loco, dos NSP do país.

Deste modo, foi possível observar que desde o ano de instituição do PNSP em 2013 até os dias atuais houve muitas 
melhorias, que certamente agregaram valor aos pacientes assistidos. No entanto, também se observa a necessidade de avanços, com maior empoderamento dos profissionais que atuam nos NSP para que, junto com todos os profissionais da instituição, alcançarem a promoção de melhorias para a segurança dos pacientes nos hospitais do Brasil.

\section{Referências}

Abu-El-Noor, N. I., Abu-El-Noor, M. K., Abuowda, Y. Z., Alfaqawi, M., \& Böttcher, B. (2019). Patient safety culture among nurses working in Palestinian governmental hospital: a pathway to a new policy. BMC health services research. 19(1), 550. 10.1186/s12913-019-4374-9

Andrade, L., Lopes, J. M., Souza, M., Filho, Vieira, R. F., Júnior, Farias, L., Santos, C., \& Gama, Z. (2018). Cultura de segurança do paciente em três hospitais brasileiros com diferentes tipos de gestão. Ciencia \& saude coletiva, 23(1), 161-172. 10.1590/1413-81232018231.24392015

Azevedo, K. C. C., Alves, A. M. P. M., Félix, Z. C., \& Viana, A. C. G. (2016). Implementation of the patient safety core in a health service. Revista enferm, UFPE. 10(12). 10.5205/reuol.9978-88449-6-ED1012201634.

Báo, A., Amestoy, S. C., Moura, G., \& Trindade, L. L. (2019). Quality indicators: tools for the management of best practices in Health. Revista brasileira de enfermagem, 72(2), 360-366. 10.1590/0034-7167-2018-0479

Becker, L. A., Rech, C. R., Hino, A., \& Reis, R. S. (2018). Evidence-based decision making and promotion of physical activity among directors of local health departments. Revista de saude publica, 52, 90. 10.11606/S1518-8787.2018052000379

Braithwaite D, Emery J, De Lusignan S, Sutton S. (2003). Using the Internet to conduct surveys of health professionals: a valid alternative? Fam Pract. 20(5), 545-51. 10.1093/fampra/cmg509.

Brasil. (1977). Portaria $n^{\circ}$. 30, de 11 de fevereiro de 1977. Aprova conceitos e definições referentes a normas e padrões para prédios e instalações destinados a Serviços de Saúde e Determina outras providência. [Internet]. Diário Oficial da Uniãohttps://www2.camara.leg.br/legin/marg/portar/1970-1979/portaria-30bsb-11-fevereiro-1977-483614-publicacaooriginal-1-ms.html.

Brasil. (2013). Portaria n. 529, de 1 de abril de 2013. Institui o Programa Nacional de Segurança do Paciente (PNSP). [Internet]. Diário Oficial da União. http://bvsms.saude.gov.br/bvs/saudelegis/gm/2013/prt0529_01_04_2013.html.

Brasil. (2013). Resolução da Diretoria Colegiada da Anvisa n ${ }^{\circ} 36$, de 25 de julho de 2013. Institui ações para a segurança do paciente em serviços de saúde e dá outras providências [Internet]. Diário Oficial da União, https://bvsms.saude.gov.br/bvs/saudelegis/anvisa/2013/rdc0036_25_07_2013.html.

Brasil. (2014). Documento de referência para o Programa Nacional de Segurança do Paciente. [Internet]. Ministério da Saúde. https://bvsms.saude.gov.br/bvs/publicacoes/documento_referencia_programa_nacional_seguranca.pdf

Brasil. (2015). Boletim Informativo Segurança do Paciente e Qualidade em Serviços de Saúde. [Internet]. Brasília. Anvisa https://www20.anvisa.gov.br/segurancadopaciente/index.php/publicacoes/item/boletimseguranca-do-paciente-e-qualidade-em-servicos-de-saude-incidentesrelacionados-a-assistencia-a-saude-2014.

Brasil. (2016). Implantação do NSP de Segurança do Paciente em Serviços de Saúde - Série Segurança do Paciente e Qualidade em Serviços de Saúde. [Internet]. Agência Nacional de Vigilância Sanitária. https://www.segurancadopaciente.com.br/wp-content/uploads/2015/09/ebook-anvisa-06-implantacao-donucleo-de-seguranca-do-paciente-em-servicos-de-saude.pdf

Brasil. (2017). Relatório de Gestão - Exercício do ano de 2017. [Internet]. Secretaria de atenção à saúde. Brasília. Ministério da Saúde. https://www.saude.gov.br/images/pdf/2018/abril/16/RELATORIO-DE-GESTAO-2017--COMPLETO-PARA-MS.pdf.

Brasil. (2019). Coordenações Estaduais dos Núcleos de Segurança do Paciente. [Internet]. Agência Nacional de Vigilância Sanitária. Brasília. Anvisa. http://portal.anvisa.gov.br/documents/33852/2961608/NSP+-+Estados/f0f60943-a28e-40ad-a773-41bbe684a88f.

Brasil. (2019). Relatório dos estados: eventos adversos - $\quad$ Brasil. $\quad$ Internet]. Brasília. Anvisa. https://www20.anvisa.gov.br/segurancadopaciente/index.php/publicacoes/category/relatorios-dos-estados.

Burstrom, L., Letterstal, A., Engstrom, M. L., Berglund, A., \& Enlund, M. (2014). The patient safety culture as perceived by staff at two different emergency departments before and after introducing a flow-oriented working model with team triage and lean principles: a repeated cross-sectional study. BMC health services research. 14, 296. 10.1186/1472-6963-14-296

Cavalcante, E., Pereira, I., Leite, M., Santos, A., \& Cavalcante, C. (2019). Implementation of patient safety centers and the healthcare-associated infections.. Revista gaucha de enfermagem, 40. 10.1590/1983-1447.2019.20180306

Halligan, M., \& Zecevic, A. (2011). Safety culture in healthcare: a review of concepts, dimensions, measures and progress. BMJ quality \& safety, 20(4), 338343. $10.1136 /$ bmjqs. 2010.040964

Institute of Medicine (US) Committee on Quality of Health Care in America, Kohn, L. T., Corrigan, J. M., \& Donaldson, M. S. (Eds.). (2000). To Err is Human: Building a Safer Health System. National Academies Press (US).

Lemos, G. C., Azevedo C., Bernardes, M. F. V. G., Ribeiro, H. C. T. C., Menezes, A. C., \& Mata, L. R. F., (2018). The patient safety culture in the scope of nursing: theoretical reflection. Revista de Enfermagem do Centro-Oeste Mineiro. 8. 10.19175/recom.v8i0.2600

Lima, K. W. S., Antunes, J. L. F., \& Silva, Z. P. (2015). Perception of managers on the use of indicators in health services. Saúde Soc. São Paulo. 24(1). 10.1590/S0104-12902015000100005 
Macedo, R. \& Bohomol, E. (2019). Organizational structure analysis of the Patient Safety Center in hospitals of the Sentinel Network. Revista Gaúcha de Enfermagem. 40. 10.1590/1983-1447.2019.20180264.

Magalhães Costa, E. A., Lobão, W. M., Ribas, C. L. M., \& Passos, N. M. (2020). Segurança do paciente em serviços de saúde: uma análise na cidade de Salvador, Bahia. Revista SOBECC. 25(1), 17-24. 10.5327/Z1414-4425202000010004"/10.5327/Z1414-4425202000010004.

McFadden, K. L., Stock, G. N., \& Gowen, C. R. (2015). Leadership, safety climate, and continuous quality improvement: impact on process quality and patient safety. Health care management review. 40(1), 24-34. 10.1097/HMR.0000000000000006

Mendes, W., Martins, M., Rozenfeld, S., \& Travassos, C. (2009). The assessment of adverse events in hospitals in Brazil. International journal for quality in health care: journal of the International Society for Quality in Health Care, 21(4), 279-284. 10.1093/intqhc/mzp022

Negri Filho, A. \& Barbosa, Z. (2014). O Papel Dos Hospitais Nas Redes De Atenção À Saúde. Elementos para pensar uma Agenda Estratégica para o SUS. Revista do Conselho Nacional de Secretários de Saúde. 20, Ano IV. http://www.conass.org.br/biblioteca/pdf/revistaconsensus_11.pdf.

Pelissari, M. (2019). CNES como instrumento de gestão e sua importância no planejamento das ações em saúde. Revista De Saúde Pública Do Paraná. 2(1), 159-165. 10.32811/25954482-2019v2n1p159

Prates, C. G., Magalhães, A., Balen, M. A., \& Moura, G. (2019). Patient safety nucleus: the pathway in a general hospital. Núcleo de segurança do paciente: o caminho das pedras em um hospital geral. Revista gaucha de enfermagem, 40. 10.1590/1983-1447.2019.20180150

Reis, C. T., Paiva, S. G., \& Sousa, P. (2018). The patient safety culture: a systematic review by characteristics of Hospital Survey on Patient Safety Culture dimensions. International Society for Quality in Health Care. 30(9), 660-677. 10.1093/intqhc/mzy080

Rennó, C. O., Ramirez, S., \& Fiad, A. (2018). Análise da implantação do Núcleo de Segurança do Paciente na rede hospitalar do município do Rio de Janeiro. Academus Revista Científica da Saúde. 3(1). https://smsrio.org/revista/index.php/reva/article/view/415.

Ricci-Cabello, I., Pons-Vigués, M., Berenguera, A., Pujol-Ribera, E., Slight, S. P., \& Valderas, J. M. (2016). Patients' perceptions and experiences of patient safety in primary care in England. Family practice. 33(5), 535-542. 10.1093/fampra/cmw046

Rocha, T., Silva, N., Barbosa, A., Amaral, P. V., Thumé, E., Rocha, J. V., Alvares, V., \& Facchini, L. A. (2018). National Registry of Health Facilities: data reliability evidence. Cadastro Nacional de Estabelecimentos de Saúde: evidências sobre a confiabilidade dos dados. Ciencia \& saude coletiva. 23(1), 229-240. $10.1590 / 1413-81232018231.16672015$

Rossaneis, M. A., Gabriel, C. S., Haddad, M. C. L., Melo, M. R. A. C., \& Bernardes, A. (2015). Indicadores de qualidade da assistência: opinião de enfermeiros gerentes de hospitais de ensino. Revista Cogitare Enferm. 20(4). https://revistas.ufpr.br/cogitare/article/view/41734/26785.

Saeed, N., Parvez, M. \& Shah, G. (2005). Effect of questionnaire, cover letter, and follow-up on non-response rate in an e-mail assisted internet survey in Pakistan. European Journal of Scientific Research. 2. 13-23. https://www.ijeat.org/wp-content/uploads/Souvenir_IJEAT_Volume-9_Issue1_October_2019.pdf

Santiago, T. H., \& Turrini, R. N. (2015). Organizational culture and climate for patient safety in Intensive Care Units. Cultura e clima organizacional para segurança do paciente em Unidades de Terapia Intensiva. Revista da Escola de Enfermagem da USP. 49, 123-130. 10.1590/S0080-623420150000700018

Santos, R. P., Soppa, F. B. F., Ruths, J. C., \& Rizzotto, M. L. F. (2019). Evaluation of the implantation of a patient safety nucleus. Revista enferm UFPE online. 13(2), 532-7. 10.5205/1981-8963-v13i2a238189

Santos, T. R., Penm, J., Baldoni, A. O., Ayres, L. R., Moles, R., \& Sanches, C. (2018). Hospital pharmacy workforce in Brazil. Human resources for health, 16(1), 1. https://doi.org/10.1186/s12960-017-0265-5

Sartor, G., da Silva, B., \& Masiero, A. (2016). Segurança do paciente em hospitais de grande porte: panorama e desafios. Cogitare Enfermagem, 21(5). $10.5380 /$ ce.v21i5.45644

Siman, A. G., Cunha, S., \& Brito, M. (2017). The practice of reporting adverse events in a teaching hospital. Revista da Escola de Enfermagem da USP. 51. $10.1590 / \mathrm{S} 1980-220 \mathrm{X} 2016045503243$

Smith, S. A., Yount, N., \& Sorra, J. (2017). Exploring relationships between hospital patient safety culture and Con sumer Reports safety scores. BMC health services research, 17(1), 143. https://doi.org/10.1186/s12913-017-2078-6 